\title{
Geometry of Entanglement Sudden Death: Explicit Examples
}

\author{
Raphael C. Drumond* and Marcelo O. Terra Cunha ${ }^{\dagger}$ \\ * Departamento de Física, ICEx, UFMG \\ Caixa Postal 702, Campus Pampulha, Belo Horizonte, MG, Brazil email \\ ${ }^{\dagger}$ Departamento de Matemática, ICEx, UFMG \\ Caixa Postal 702, Campus Pampulha, Belo Horizonte, MG, Brazil email
}

\begin{abstract}
In open quantum systems, entanglement can vanish faster than coherence. This phenomenon is usually called sudden death of entanglement. In [M. O. Terra Cunha, New J. Phys. 9, 237 (2007)] a geometrical explanation was offered and a classification of all possible scenarios was given. Some classes were exemplified, but it was still an open question whether there were examples for the other ones. This was solved in [R. C. Drumond and M. O. Terra Cunha, arXiv:0809.4445v1]. Here we briefly review the problem, state our results in a precise way, discuss the generality of the approach, and add some speculative desirable generalizations.
\end{abstract}

Keywords: Entanglement Sudden Death; Geometry of Quantum States PACS: $02.50 . \mathrm{Cw}, 03.65 . \mathrm{Yz}, 03.67 . \mathrm{Mn}$

\section{INTRODUCTION}

Entanglement is a valuable property of some quantum states. In the last few years attention was called to its timeevolution in the context of quantum open systems [1]. In special, it has been considered a counterintuitive fact that for some systems and initial states, entanglement can vanish in finite time, while coherences only disappear asymptotically. Intuition was put in the right perspective in the paper [2], with a geometrical picture of entanglement dynamics given in terms of the position of asymptotic states with respect to the important subsets of entangled and separable quantum states. Despite the classification problem be completely characterized in this picture, the existence of examples for some classes stayed unclear until the recent reference [3].

The present contribution will take the opportunity to state this results in a more general form, to discuss the deepness and also to clarify a new probabilistic approach to the question: given a quantum system what will be the typical behavior of entanglement? Finally, we make some considerations on the more ambitious project, related to the so called Palis Conjecture, discussing what would be the behavior of a typical open quantum system.

\section{STATEMENT OF THE RESULT}

For the sake of clarity, we will state our main result as a Theorem[1]. Despite the fact that the majority of the discussions be made using the two-qubit context, the results are much more general, as we may discuss latter on in this paper.

Let us prepare the context first. We call $D$ the set of all quantum states (in the sense of density operators) of a given system. This closed convex set has a (closed and convex) subset $S$, of separable quantum states, and the complement $E=D \backslash S$ is made of the entangled states. As we are concerned with time varying quantum states (which can be considered as curves on $D$ ), it becomes important to consider a trichotomy $\{$ int $S, \partial S, E\}$, where int $S$ denotes the interior of the set $S$, which sometimes we call deeply separable state 2 , $\partial S$ is the boundary of the set $S$ (relative to $D$ ) and we remember that $E$ can be considered as an open set relative to $D$ (i.e. any point of $E$ has a neighborhood of points of $D$ totally contained in $E$ ).

\footnotetext{
${ }^{1}$ Pretty much inspired on Prof. I. Volovich, who would usually ask to a contributor: "what is your theorem?"

${ }^{2}$ Here we restrict our attention to the finite dimensional case, where it is known[4] that $S$ has positive measure when $D$ is given the normalized measure 1 .
} 
When a quantum system evolves in contact with a reservoir, it is typical to have a set (unitary or not) of asymptotic states. Let us denote this set by $A$. The behavior of the time evolution of entanglement can be classified and characterized in terms of the relative position of such sets. The situation can vary depending on the cardinality of $A$ (denoted $|A|$ ). The results are summarized in the following classification and existence theorem:

Theorem With the above definitions of $D, S$, int $S, \partial S, E$ and $A$, any quantum open system with linear dynamics and nontrivial set $A$ of asymptotic states belongs to one, and only one, of the following classes

1. $A=\left\{\rho_{a}\right\}$ with $\rho_{a} \in$ int $S$;

2. $A=\left\{\rho_{a}\right\}$ with $\rho_{a} \in \partial S$;

3. $A=\left\{\rho_{a}\right\}$ with $\rho_{a} \in E$;

4. $|A|>1$ with $A \subset$ int $S$;

5. $|A|>1$ with $A \cap \partial S \neq \emptyset$;

6. $|A|>1$ with $A \subset E$.

Moreover, examples of quantum systems can be obtained for each of these classes.

\section{IDEA OF THE PROOF}

The proof of the classification part of the Theorem is simple. If $A$ is nontrivial, it must be unitary or larger. The first three classes correspond to the unitary case, and each class corresponds to the only element $\rho_{a}$ belonging to one of the three mutually exclusive alternatives int $S, \partial S$, and $E$. For the larger $A$ situation it is important to recognize that linearity of quantum evolution implies a convex (hence connected) $A$. The first question is whether $A \cap \partial S$ is empty. In negative case, the system belongs to the fifth class, in affirmative case, in order to avoid $\partial S, A$ must be contained in one of the alternatives: int $S$ or $E$.

The existence part is constructive. We explicitly give examples for each class, as can be checked on ref. [3]. It is interesting to note that for the construction of the examples it was sufficient to consider two qubits; however, it was necessary to allow non-autonomous systems.

\section{INTERPRETATION AND GENERALITY}

First of all, the geometric interpretation of entanglement time evolution demystify the phenomenon of sudden death of entanglement. If $A \subset$ int $S$, any initial state needs to lose entanglement in finite time in order to approach the asymptotic set.

Moreover, the classification scheme also allows for the counterpart of this phenomenon, whenever $A \subset E$. In such a case, irrespective of the initial presence of entanglement, after a finite amount of time there will be some entanglement.

The more interesting situations are the intermediate ones. The situation $A=\left\{\rho_{a}\right\}, \rho_{a} \in \partial S$ has already received special attention in the literature [5], with the simple and interesting example of two independent qubits spontaneous decaying. Depending on the initial state (even with the same amount of initial entanglement) entanglement can die in finite time or only asymptotically. This is vary natural, since $\rho_{a}$ can be approached both, by $\rho(t) \in S$ ("sudden death") or $\rho(t) \in E$ ("eternal life"). In such cases $(A \cap \partial S \neq \emptyset)$, an important refinement of this study can be made case by case: if both situations are possible, which one is prevalent? In what proportions do they happen? This can be well defined [3] in terms of the measure of the sets of initial states with each foreseen fate.

It must be clear that, despite the fact that much of our discussion focus on two-qubit examples for simplicity, our results apply as well for higher dimensionality and for the multipartite context, when we can define more than one kind of entanglement. In this case, $S$ must be considered as the states which do not have the specific kind of entanglement one wants to consider and all the previous reasoning apply. Of course, quantitative statements vary. Also, it is interesting to recognize that for the multipartite scenario, one can have a hierarchy of entanglements, and very interesting pictures can be draw including cases when all entanglements vanish together [6] or the more general situation when the family lose one member per time.

\footnotetext{
${ }^{3}$ Unitary here, and throughout the paper, means with only one element.
} 


\section{MORE SPECULATIVE COMMENTS}

An even more ambitious plan wouldbe to relate this study of time evolution of entanglement to the so called Palis Conjecture on dynamical systems [7]. In a precise way, Palis enunciate that the vast majority of hyperbolic dynamical systems has a finite number of attractors, whose basins gives a set of total measure in the phase space.

Here, as quantum mechanical evolutions are simpler than general hyperbolic dynamics on manifolds, we could dream of parameterizing the set of possible evolutions for a well defined system (e.g. two qubits), and of finding a

meaningful measure on such a set, in order to give quantitative answers to the question what is the typical behavior of a quantum system?

\section{ACKNOWLEDGMENTS}

MOTC wants to thank the organizers for the special meeting they hold. Fapemig is acknowledged for founding the research and the travel. RCD thanks financial support from CNPq.

\section{REFERENCES}

1. K. Życzkowski et al, Phys. Rev. A 65, 012101 (2001); L. Diósi, Lect. Notes Phys. 622, 157 (2003); P. J. Dodd and J. J. Halliwell, Phys. Rev. A 69, 052105 (2004); T. Yu and J. H. Eberly, Phys. Rev. B 66, 193306 (2002); Opt. Cummun. 264, 393 (2006).

2. M. O. Terra Cunha, New J. Phys. 9, 237 (2007).

3. R. C. Drumond and M. O. Terra Cunha, arXiv:0809.4445v1

4. I. Bengtsson and K. Życzkowski, Geometry of Quantum States: An introduction to quantum entnaglement, Cambridge University Press (2006).

5. M França Santos et al, Phys. Rev. A 73, 040305 (2006)

6. L. Aolita et al, Phys. Rev. Lett 100, 080501 (2008).

7. J. Palis, Annales de l'Institut Henri Poincare, Non Linear Analysis 22, 485 (2005). 